not name some study benefit. 38\% were unsure/did not know the total number of visits study required of them. $74 \%$ knew the duration of the study. DISCUSSION/SIGNIFICANCE OF IMPACT: Extended discussion and more time on one-one by the study teams in this CTSA tend to increase trust. This approach has been reported to be most effective in improving participant understanding of informed consent process and may result in the positive experience.

4298

Prediction models for pulmonary tuberculosis treatment outcomes: a systematic review

Lauren Saag Peetluk, $\mathrm{MPH}^{1}$, Felipe Ridolfi, Valeria Rolla, and

Timothy Sterling

${ }^{1}$ Vanderbilt University Medical Center

OBJECTIVES/GOALS: Many clinical prediction models have been developed to guide tuberculosis (TB) treatment, but their results and methods have not been formally evaluated. We aimed to identify and synthesize existing models for predicting TB treatment outcomes, including bias and applicability assessment. METHODS/STUDY POPULATION: Our review will adhere to methods that developed specifically for systematic reviews of prediction model studies. We will search PubMed, Embase, Web of Science, and Google Scholar (first 200 citations) to identify studies that internally and/or externally validate a model for TB treatment outcomes (defined as one or multiple of cure, treatment completion, death, treatment failure, relapse, default, and lost to follow-up). Study screening, data extraction, and bias assessment will be conducted independently by two reviewers with a third party to resolve discrepancies. Study quality will be assessed using the Prediction model Risk Of Bias Assessment Tool (PROBAST). RESULTS/ANTICIPATED RESULTS: Our search strategy yielded 6,242 articles in PubMed, 10,585 in Embase, 10,511 in Web of Science, and 200 from Google Scholar, totaling 27,538 articles. After de-duplication, 14,029 articles remain. After screening titles, abstracts, and full-text, we will extract data from relevant studies, including publication details, study characteristics, methods, and results. Data will be summarized with narrative review and in detailed tables with descriptive statistics. We anticipate finding disparate outcome definitions, contrasting predictors across models, and high risk of bias in methods. Meta-analysis of performance measures for model validation studies will be performed if possible. DISCUSSION/SIGNIFICANCE OF IMPACT: TB outcome prediction models are important but existing ones have not been rigorously evaluated. This systematic review will synthesize TB outcome prediction models and serve as guidance to future studies that aim to use or develop TB outcome prediction models.

4084

Predictors of mortality among non-severe hemophilia A patients in the United States

Ming Y Lim ${ }^{1}$, Dunlei Cheng ${ }^{2}$, and Nigel Key ${ }^{3}$

${ }^{1}$ University of Utah School of Medicine; ${ }^{2}$ ATHN; ${ }^{3}$ University of North Carolina

OBJECTIVES/GOALS: To determine predictors of mortality in nonsevere hemophilia A (NSHA) patients. METHODS/STUDY POPULATION: The ATHNdataset was used to identify NSHA patients who have authorized the sharing of their demographic and clinical information for research. Factors examined included race, ethnicity, hemophilia severity, Hepatitis B, Hepatitis C and HIV infections. A mortality rate was calculated for each factors examined. The relative risk of death between patients in different categories of the factors was assessed by using the ratio of these mortality rates. To adjust for the effects of all of the studied factors with mortality, a multivariate analysis was performed using logistic regression. All hypothesis testing was two-tailed, with a significance level of .05. RESULTS/ ANTICIPATED RESULTS: A total of 6,606 NSHA patients were followed for an average of 8.5 years. During 56,064 person years of observation, $136(2.1 \%)$ NSHA patients died; $20 \%$ of deaths were malignancy-related. Mortality rates were similar across racial group. Hispanic patients were $60 \%$ less likely to die than non-Hispanic patients $(\mathrm{p}=0.006)$. Patients with Hepatitis C infection and HIV infection were 7 times as likely to die compared to those without infections $(\mathrm{p}<0.001)$. After adjusting for the effects of all examined factors in a multivariate analysis, patients with hepatitis $\mathrm{C}$ and HIV infection remain significantly associated with increased mortality at 6.1 times and 3.6 times the risk, respectively. DISCUSSION/SIGNIFICANCE OF IMPACT: Despite significant improvement in the therapeutic approaches for infectious diseases, Hepatitis C and HIV infections remain strong predictors of mortality in this NSHA cohort. CONFLICT OF INTEREST DESCRIPTION: N/A.

4065

\section{Preferences, Expectancies, and Stigma among Treatment Seeking Combat PTSD Patients}

John Moring ${ }^{1}$, Alan Peterson ${ }^{2}$, Casey Straud ${ }^{2}$, Jim Mintz ${ }^{2}$, Paul Nabity $^{2}$, Lindsay Bira ${ }^{2}$, Stacey Young-McCaughan ${ }^{2}$, Willie Hale ${ }^{3}$, Donald McGeary ${ }^{2}$, and Patricia Resick ${ }^{4}$

${ }^{1}$ University of Texas Health Science Center San Antonio; ${ }^{2}$ UT Health San Antonio; ${ }^{3}$ University of Texas at San Antonio; ${ }^{4}$ Duke University Medical Center

OBJECTIVES/GOALS: Cognitive Processing Therapy (CPT) is a cognitive behavioral treatment for posttraumatic stress disorder (PTSD). CPT is effective in treating combat-related PTSD among Veterans and active duty service members. It is unknown whether improvement in PTSD is related to accommodation of patient preference of the modality of therapy, such as in-office, telehealth, and in-home settings. An equipoise-stratified randomization design allows for complete randomization of participants who are interested and eligible for all three treatment arms. It also allows participants to reject one treatment arm if they are not interested or eligible. Participants who elect to opt out of one arm are randomized to one of the two remaining treatment arms. The primary aim of this study was to evaluate differences in patient satisfaction, treatment stigma beliefs, and credibility beliefs based on patient treatment modality preference. The second aim of this study was to examine if baseline satisfaction, stigma beliefs, and credibility beliefs predicted PTSD treatment outcomes. METHODS/STUDY POPULATION: Active duty service members and veterans with PTSD $(\mathrm{N}=123)$ were randomized to one of three arms using an equipoise stratified randomization. Participants underwent diagnostic interviews for PTSD at baseline and post-treatment and completed self-report measures of satisfaction, stigma, credibility and expectancies of therapy. RESULTS/ANTICIPATED RESULTS: A series of ANOVAs indicated that there were group differences on patient stigma beliefs regarding mental health, $\mathrm{F}=5.61, \mathrm{p}=.001$, and therapist credibility, $\mathrm{F}=5.11, \mathrm{p}=.002$. Post hoc analyses revealed that participants who did not opt of any treatment arm demonstrated lower levels of 